Pedagogía y Saberes No. 46

Universidad Pedagógica Nacional

Facultad de Educación. 2017, pp. 45-54

\title{
La mayor disrupción posible en la historia de la pedagogía moderna: Ivan Illich
}

Artículo de investigación

The biggest disruption possible in the History of Modern

Pedagogy: Ivan Illich

A maior irrupção possível na história da pedagogia moderna: Ivan Illich

Mariano Narodowski*

Mayra Botta**

Doctor en educación de la Universidade Estadual de Campinas (UNICAMP), profesor Titular de la Universidad Torcuato Di Tella. E-mail: mnarodowski@utdt.edu

Perfil ORCID: http://orcid.org/0000-0002-3122-052X

** Licenciada en Psicopedagogía, asistente de Investigación, Universidad Torcuato Di Tella. E-mail: mayra.botta@gmail.com Perfil ORCID: http://orcid.org/0000-0002-3230-782X 


\title{
Resumen
}

Este artículo analiza el pensamiento del pedagogo Ivan Illich en el devenir de la pedagogía moderna. En primer lugar, muestra el consenso en la literatura académica internacional respecto de las instituciones escolares y postula la posibilidad de su contingencia y su sustitución. Se muestra cómo los textos de Illich configuran una transgresión a los límites epistémicos de la pedagogía moderna, expresando en la posibilidad de desescolarización el máximo nivel de disrupción respecto de los dispositivos pedagógicos. Se analizan las propuestas de Illich en un escenario desjerarquizado y prefigurativo y se resalta el valor de sus hipótesis ambiciosas y provocativas. Finalmente, se muestra la fortaleza y actualidad illichiana frente a un futuro donde la escolarización moderna tiende a ser cuestionada.

\section{Palabras clave}

Ivan Illich, desescolarización, pedagogía, dispositivos, ideal pansófico.

\begin{abstract}
This paper studies Ivan Illich's pedagogical thinking on the becoming of modern pedagogy. First, it shows the international consensus in academic literature concerning educational institutions, and states the possibility of their contingency and substitution. The paper displays how Illich's texts constitute a transgression of the epistemic boundaries of modern pedagogy, expressing through the possibility of deschooling the ultimate level of disruption regarding pedagogical devices. Illich's proposals are analyzed under a prefigurative and nonhierarchical light, emphasizing the value of his ambitious and provocative hypotheses. Finally, it shows the Illichian strength and topicality regarding a future where modern schooling tends to be questioned.
\end{abstract}

\section{Key words}

Ivan Illich, deschooling, pedagogy, devices, Pansophia.

\section{Resumo}

0 artigo analisa o pensamento de Ivan Illich na evolução da pedagogia moderna. Em primeiro lugar, mostra o consenso na literatura acadêmica internacional sobre as instituições de ensino e postula a possibilidade da sua contingência e substituição. 0 estudo demonstra como os textos de Illich constituem uma transgressão dos limites epistêmicos da pedagogia moderna, expressando a possibilidade de desescolarização: o mais alto nível de perturbação sobre dispositivos pedagógicos. Analisam-se as propostas de Illich num cenário prefigurativo e desierarquizado, destacando o valor das suas hipóteses ambiciosas e provocativas. Finalmente, mostra-se a força e atualidade Illichiana frente a um futuro onde a escolaridade moderna tende a ser questionada.

\section{Palavras chave}

Ivan Illich, desescolarização, pedagogia, dispositivos, ideal pansófico. 


\section{Introducción}

Desde hace décadas, sabemos que la institución escolar es un epifenómeno de la modernidad.

\section{Hamilton, 2013 (traducción propia)}

A pesar de que estamos acostumbrados a la idea de que en nuestras sociedades las personas deben educarse en escuelas, como si esto fuera un mandato de la naturaleza, la investigación ha demostrado sobradamente que el ser humano transcurrió la casi totalidad de su historia como especie transmitiendo saberes sin escuelas y que las mismas se originan recién en el siglo xvii europeo y se difunden globalmente solo desde la segunda mitad del siglo xx, hace unos pocos años. Más todavía, hacia el año 2015, y en las regiones más pobres del mundo, ingentes masas poblacionales aún no accedían a la educación escolar o transitaban por ella durante tiempos exiguos (Valle, Normandeau y González, 2015).

Esta institución escolar de la modernidad mantiene, a pesar de matices nacionales o territoriales y de ciertas diferencias temporales, una matriz común que la hace inconfundible: ya sea una choza en un aislado y empobrecido paraje latinoamericano o una construcción tecnológicamente avanzada en una muy desarrollada ciudad europea, el contorno de lo escolar con la disposición de sus espacios y la organización de sus tiempos comparte ese entramado común que cobra sentido inmediato e indiscutible para cualquier habitante de nuestra era (Hamilton, 2013; Narodowski, 1994).

Pero también sabemos que la matriz escolar no está constituida solamente por la dispersión topológica o la expansión cronológica. Las escuelas de la modernidad anidan invariantes en las gramáticas y en las relaciones sociales (e intergeneracionales) que conforman dispositivos coagulados en su práctica histórica, los que producen un conjunto de efectos semejantes, más o menos estables, más o menos previsibles (Cuban, 1990).

Como hemos pretendido demostrar en varios estudios, la idea de alumno como efecto de la infantilización de una parte de la población, la instrucción simultánea en la que el docente ocupa el lugar del saber, la sala de aula y la simultaneidad sistémica que organiza escuelas bajo la jerarquía de una instancia supraescolar (el Estado) que determina un currículo unificado son algunos de los dispositivos de las instituciones escolares que delimitan no solo los confines de lo escolarizable (es decir, le ponen una barrera a la usualmente omnipotente voluntad racional de los educadores) sino que también marcan la finitud de lo pensable: ambos aspectos ponen coto a aquello que se puede siquiera imaginar respecto de lo que es la escuela (y a la larga delo que es la educación) y aquello que estas instituciones pueden (y no pueden) concretar (Narodowski, 1994, 1999).

El escenario escolar, lo sabemos desde los estudios de Margaret Mead, solo es posible en el contexto de una cultura posfigurativa, de cambios sociales relativamente lentos que hacen que la antigüedad en la vida social acumule sabiduría, por lo que los más antiguos (los adultos, los ancianos) deben propiciar ejemplos para que los más jóvenes sigan ese camino y se vuelvan, con el transcurrir del tiempo, portadores ellos mismos de esa sabiduría (Mead, 1970).

El conjunto escolar de la modernidad es, por lo tanto, posfigurativo; los embajadores de la tradición(los padres, los sacerdotes, y especialmente los maestros de las escuelas)ocupan el lugar jerarquizado del saber y lo transmiten a los recién llegados a la cultura. La clásica definición de educación de Emile Durkheim (1973), que la pedagogía ha adoptado como un rezo, se ajusta perfectamente a esta situación: la educación es la transmisión de saberes de las generaciones más viejas a las más jóvenes. Siguiendo este mandato, los dispositivos escolares se articulan a favor de una linealidad vertical, disciplinaria. La escuela de la modernidad es la de un tiempo en el que saberes ordenados y organizados eran transmitidos, monopólicamente, por una institución en la que adultos ordenaban a infancias que obedecían.

Pero este conocimiento riguroso, preciso, analítico del ejercicio de los poderes que escolarizan tiene una consecuencia que no siempre se tiene en cuenta: si la escuela ha sido un epifenómeno de la modernidad, si la escuela es un emergente temporal cuyo inicio histórico y cuyas coordenadas sociales son perfectamente identificables, no hay motivo para no suponerla como una contingencia, como otro artefacto capaz de declinar irremediablemente y ser sustituido por cualquier otro (Martínez y Narodowski, 1986).

Es notorio cómo esta idea suele ser poco aceptada entre los educadores, incluso entre aquellos que han estudiado y hasta denunciado la lógica más dura de los procesos de conformación de lo escolar. Desarrollarla, desplegarla, jugarla o apenas imaginarla provoca un vértigo singular: enfrentarse a la sola idea de una sociedad desescolarizada es como pararse en la baranda del balcón más alto de un rascacielos para intentar piruetas que, sabemos, nos pueden llevar al fin.

Este es el lugar del pensamiento de Ivan Illich en la historia de la pedagogía reciente. Un lugar provocador, irreverente, desafiante: se trata de transgredir la finitud de lo pedagógicamente pensable y así desafiar las leyes de la gravedad de la pedagogía de la modernidad. 
Es el acto revolucionario por antonomasia al que los pedagogos se resisten más que a nada: saltar es aceptar la posibilidad de la propia desaparición.

Porque la certeza del conocimiento riguroso y preciso de los dispositivos de la escolaridad nos alerta sobre algo más oscuro y misterioso: desde Lyotard (1979) sabemos que nuestros conocimientos sobre el poder, la descripción pormenorizada de su ejercicio y la enumeración meticulosa de sus efectos (tan difundida en el canon académico al punto que ya se han convertido en lugar común: tema de estudio universitario, pregunta en un examen, aburrido tema de tesis) anticipan que esos poderes han dejado de operar, o al menos ya no lo hacen con la misma virulencia y presteza de antaño: han perdido su eficacia inaugural basada, entre otras cosas, en el misterio de su sujeción.

Por otro lado, la hegemonía de la cultura posfigurativa también va llegando a su fin (Narodowski, 2016). Nuestra cultura contemporánea es de orden prefigurativo y apunta a cambios sociales y tecnológicos tan violentos que las diferencias jerárquicas intergeneracionales van diluyéndose a favor de organizaciones horizontales en las que la escuela pierde su lugar monopólico y los adultos su pedestal originario: al contrario, en nuestro mundo sin adultos la vejez es sospechosa y la palabra de la autoridad de los mayores siempre sometida al escrutinio por su posible obsolescencia.

En el pliegue de esta época en la que la escuela no termina de morir y lo radicalmente nuevo no termina de nacer, en esa línea tensa de grosor ínfimo que conforma el ángulo donde se resultan mutuamente lo viejo y lo nuevo, es Ivan Illich quien consigue comprender la naturaleza definitiva del deterioro escolar, su carácter ineluctable. Y su imaginación, preñada del vértigo arremolinado y angustiante de la ilusión de un fin, dio luz al texto que consigue transgredir la finitud de lo pedagógicamente pensable.

\section{La mayor disrupción posible de la pedagogía}

Para Illich (1985), las personas ya no se deben aglutinar en establecimientos educacionales especializados en la educación de niños y jóvenes, sino que habrán de organizarse en redes configuradas por el interés, por medio de las cuales los diversos actores sociales procuran su acercamiento al saber en forma voluntaria, focalizada, flexible y electiva.

Este planteo se sitúa en el centro de la crítica al carácter disciplinante y uniformizador de las instituciones escolares de la modernidad, y del docente adulto que encarna un saber en forma socialmente autoritaria y epistemológicamente ortodoxa. En congruencia con los escritos de Paulo Freire (2005) de los años sesenta, Illich desafía la necesidad de asimetrías en la transmisión de saberes: de simetrías individuales (lugares fijos de enseñantes y enseñados) y simetrías colectivas: sistemas escolares estatales, obligatorios y jerárquicos.

El enfoque no implica una reformulación del ideal pansófico de la modernidad, el viejo "enseñar todo a todos", la utopía igualitarista instalada desde los escritos del siglo xvii de Jan Amos Comenius (1986) sino, al contrario, su puesta a punto en términos de época. Illich lo reafirma con crudeza: "El que todos tengan iguales oportunidades de educarse es una meta deseable y factible, pero identificar con ello la escolaridad obligatoria es confundir la salvación con la iglesia" (1985, p. 28).

Illich apunta directamente contra la institución escolar como organización coercitiva de la producción y distribución de saberes -que tiene en los sistemas escolares nacionales su máxima expresión- por su carácter disciplinante, pero sobre todo ineficaz, en la medida en que no avanza ni siquiera en los modestos objetivos que se plantea. Por eso, en un giro inesperado, en ese vértigo transgresor, pretende sustituir tal sistema por un conjunto de espacios voluntarios de articulación social (una o varias redes) que se anudan para dar paso al acceso irrestricto al saber; una pansofía ahora horizontalizada en redes en las que apenas se diferencian aquellos que "poseen" el saber respecto de aquellos que no lo detentan, suponiendo que todos son portadores de algún saber de interés para los demás, otra idea marcadamente freireana.

Vale recordar que esta propuesta, surgida en los inicios de los años setenta, se inscribía en una corriente de fuerte cuestionamiento a las instituciones escolares: Freire e Illich fueron, posiblemente, los últimos pedagogos "críticos" de la modernidad, los últimos dinosaurios de una época a punto de eclosionar.

Contemporáneamente a Illich, y antes que él, Everrett Reimer, así como los libros y artículos de Paulo Freire interpelaban cuestiones análogas y algunos sociólogos franceses como Louis Althusser, Pierre Bourdieu, Christian Baudelot o Roger Establet marcaban un intenso tono crítico a lo que denominaban mecanismos de reproducción de las relaciones capitalistas de producción que las escuelas y los sistemas educativos llevaban a cabo. Pero a diferencia de los pedagogos críticos, Illich no pretende cambiar la escuela, renovarla, mejorarla y ni siquiera emanciparla. Quiere sustituirla, traspasar los límites de lo pedagógicamente pensable, ejercer la máxima disrupción posible. 
También en la línea freireana, en los argumentos de Ivan Illich se sublima una crítica no siempre reconocida al rol de la autoridad adulta del maestro en los sistemas escolares conocidos. Es verdad que en el modelo propuesto por Illich no se destituye la figura del maestro, como lo veremos. Lo que sí habría de desaparecer, o al menos se diluiría hasta hacerla poco relevante, es la centralidad del maestro como ocupante del lugar monopólico del saber, centralidad que en la propuesta illicheana queda relegada en virtud de esta suerte de aplanamiento desjerarquizado en el que las personas toman lugar horizontalmente para proveer y recibir conocimientos de acuerdo a sus intereses, su conveniencia, sus opciones; sin sujetarse a la lógica asimétricamente inconmovible, propia del proceso de escolarización de la sociedad. En otras palabras, Illich le apuesta a un magisterio sin asimetrías, o en el mejor de los casos con asimetrías contingentes, que duran lo que el interés por determinado saber.

\section{Tramas de aprendizaje}

En el capítulo "Tramas de aprendizaje", Ivan Illich (1985) plantea la necesidad de generar nuevas estructuras relacionales que permitan a cada uno definir su propio aprendizaje y contribuir al de otros, un "nuevo estilo de relación educativa entre el hombre y su medio" (p. 102) como alternativa a las instituciones educativas de la modernidad. Esto implica un cambio en el estilo de consumo de los recursos de aprendizaje y la necesidad de generar nuevos vínculos para su apropiación, orientados al autoaprendizaje.

Este punto de vista impacta por debajo de la línea de flotación de la escuela de la modernidad puesto que se posicionan-en contraposición a sus dispositivos pedagógicos y a la centralidad de la autoridad docente-, el currículo unificado y la simultaneidad sistémica. Para Illich, las instituciones educativas crean dependencia y el "hábito del consumo de servicios y producción enajenante" (1985, p. 104).

Los objetivos de las nuevas y desescolarizadas instituciones educativas que plantea Illich son tres: (1) propiciar el acceso a los recursos educativos a lo largo de toda la vida,y no solamente en una fase infantilizada, (2) facilitar el encuentro de aquellos que quieran compartir lo que saben con los que quieran aprender, removiendo jerarquías burocráticas acerca del saber y sus certificaciones y (3) generar oportunidades de presentar y debatir públicamente sus argumentos.

Estas instituciones debieran ser canales de acceso sin restricciones (sociales, culturales, etarias), ordenados según su especificidad, para que todos tengan acceso a ellos. A este esquema se le denomina trama de oportunidades, según Illich, el término que mejor define una estructura de acceso recíproco que genere igualdad de oportunidades para enseñar y para aprender; una red que posibilite la libre expresión, tanto para registrar y preservar como para difundir opiniones.

El escenario de esta reciprocidad proviene de otro concepto illicheano central: la convivencialidad (Illich, 1974). Las máquinas, las tecnologías, las escuelas, no pueden permanecer en la centralidad de la vida social sino al contrario, son las personas las que deben hacer un uso de ellas en la medida en que estén a su altura, su perfil y sus objetivos. La convivencialidad supone una suerte de altruismo activo que desactiva las formas típicas de enajenación y permite una colaboración franca, genuina, desprejuiciada, entre todos los poseedores de conocimiento.

Por eso, el planteamiento de estas nuevas instituciones educativas parte de la pregunta acerca de qué cosas y con quién debería contactarse aquel que quiere aprender algo, en lugar de preguntarse qué debería aprender alguien con independencia de su identidad y su interés. Si en una organización convivencial se parte desde la persona que aprende, y para aprender se requiere tanto de información como de "reacción crítica" (Illich, 1985, p. 109) acerca de cómo utilizar esa información. Esa reacción crítica es dada por otras personas, que pueden ser iguales o mayores. Los iguales son los compañeros de aprendizaje, que comparten intereses comunes, mientras que los mayores son aquellas personas con una experiencia superior, que pueden convertirse en "guías" mediante el asesoramiento acerca de qué destreza aprender, qué método usar, qué compañía buscar, etc. Nótese que estas guías no remiten necesariamente a un entramado posfigurativo de dominio jerárquico o a una dimensión de autoridad natural del mundo adulto, propios de las escuelas de la modernidad.

De alguna manera, la argumentación de Illich acerca de la organización de estas nuevas instituciones educativas se apoya en la forma en que las personas aprenden en contextos naturales, y lo ejemplifica de la siguiente manera:

El niño crece en un mundo de cosas, rodeado de personas que sirven de modelos para habilidades y valores. Encuentra seres como él, sus iguales, que le incitan a discutir, a competir, a cooperar, a entender; y si el niño es afortunado, se ve expuesto a la confrontación o a la crítica de un mayor experimentado que realmente se preocupe. Cosas, modelos, iguales y mayores son cuatro recursos cada uno de los cuales requiere un tipo diferente de ordenamiento para asegurar que todos tengan acceso a él. (Illich, 1985, p. 107). 
Para lograr el acceso a los recursos necesarios para aprender, se requiere de nuevas estructuras de relación, lo que Illich denomina estructuras tramadas, bajo las cuales puedan organizarse los recursos, de manera tal que puedan ayudar al que aprende a definir sus propias metas de aprendizaje y alcanzarlas.

Los recursos educacionales suelen rotularse según las metas curriculares de los educadores. Propongo hacer lo contrario, y rotular cuatro enfoques diferentes que permitan al estudiante conseguir el acceso a cualquier recurso educativo que pueda ayudarle a definir y lograr sus propias metas. (Illich, 1985, p. 110).

Estos cuatro enfoques, o "canales" organizativos de los recursos educativos, los denominó: (1) servicios de referencia respecto de objetos educativos, (2) lonjas de habilidades, (3) servicio de búsqueda de compañero, y (4) servicios de referencia respecto de educadores independientes.

Los servicios de referencia respecto de objetos educativos implican el acceso no escolar a los recursos de aprendizaje, los cuales podrían almacenarse en lugares públicos como por ejemplo en bibliotecas, laboratorios, museos, fábricas. El punto de partida de este enfoque es que lo escolar mantiene alejado de manera artificial a las personas de estos recursos debido a que los materiales educativos fueron monopolizados por las escuelas, y rotulados como instrumentos educativos que los apartan del uso cotidiano, y se encuentran al servicio del currículo. Por ejemplo, el uso de mapas, enciclopedias y de los laboratorios se realiza cuando el programa educativo dice que hay que utilizarlos.

\section{[...] los materiales educativos han sido monopoli- zados por la escuela. [...] El profesor es celoso del libro de texto al que define como su instrumento profesional. El estudiante puede llegar a odiar el laboratorio porque lo asocia con tareas escolares. El administrador racionaliza su actitud protectora hacia la biblioteca como una defensa de un instru- mental público costoso contra quienes quisieran jugar con ella más bien que aprender. (Illich, 1985, p. 112).}

Revertir eso, según este autor, requiere, necesariamente, revertir tanto el espacio físico donde se almacenan -para que estén disponibles- como su alcance para el aprendizaje autodirigido, porque cuando se utilizan determinados elementos por fuera del contexto escolar dan realmente oportunidades para lograr aprendizajes superadores. De esta manera, propone desescolarizar los artefactos de educación, y situarlos al alcance de todos, niños y adultos, en una suerte de "ciudad abierta al pueblo".
En una ciudad abierta al pueblo, los materiales de enseñanza que ahora se encierran en almacenes y laboratorios podrían diseminarse en depósitos abiertos a la calle y gestionados de manera independiente, que los adultos y los niños pudiesen visitar sin peligro de ser atropellados. Si las metas de la educación ya no estuviesen dominadas por las escuelas y los maestros de escuela, el mercado para los aprendices sería mucho más variado y la definición de "artefactos educativos" sería menos restrictiva. (Illich, 1985, p. 117).

Por tanto, los servicios de referencia de objetos de aprendizajes pueden definirse como espacios abiertos al público y centros de aprendizaje abiertos a la calle que posibiliten, como ejemplifica Illich, el acceso a materiales audiovisuales en circuitos cerrados, acceso a muestras y objetos de museo, laboratorios fotográficos, imprentas, entre otros, que permitan aprender haciendo, y quienes coordinen esos espacios tendrían un rol más parecido al de un bibliotecario que al de un docente.

En cambio, las lonjas de habilidades o el canal de profesores de habilidades debiera organizarse de manera diferente al canal de objetos, puesto que refiere a personas que quieren y acuerdan enseñarle algo a alguien, y que pueden definir o limitar el espacio, el tiempo y la metodología de la enseñanza.

Las lonjas de habilidad consisten en brindarle a una persona la posibilidad de hacer una lista de sus habilidades y condiciones, las cuales estén dispuestas a enseñar a aquellos que quieran aprenderlas. Son fuentes de enseñanza, y modelos para aprender, o como lo define Illich (1985): “Un modelo de habilidad es una persona que posee una habilidad y está dispuesta a demostrar su práctica" (p. 121).

Básicamente, este canal consiste en dar la oportunidad de enseñar algo a alguien, y ofrecer espacios y opciones para quienes quieran aprender, según sus intereses, en el tiempo que deseen, sin depender de la gradualidad de un sistema escolar. Para su funcionamiento, sería necesario un organismo que facilitara la información (listas de personas y habilidades capaz de conectar al que quiere enseñar con el que quiere aprender.

Illich reconoce que determinadas habilidades podrían aprenderse a través de objetos, como pueden ser cintas grabadas (audio o video), pero que es esperable que ciertas habilidades, especialmente las comunicacionales, sigan demandando demostraciones personales. Para ilustrarlo, presenta el siguiente ejemplo:

En nuestro Centro, en Cuernavaca, han aprendido castellano unos diez mil adultos -en su mayoría personas muy motivadas que deseaban obtener una 
cuasi-fluidez en un segundo idioma-. Cuando se les plantea la elección entre una instrucción cuidadosamente programada en un laboratorio de idiomas o sesiones rutinarias con otros dos estudiantes y una persona cuyo idioma nativo es el español y que se ciñe a un rutina rígida, la mayoría prefiere la segunda alternativa. (Illich, 1985, p. 122).

También reconoce la importancia de que aquellos que enseñan algo deben ser capaces de detectar dificultades de aprendizaje, así como motivar a sus aprendices para alcanzar los objetivos. Es decir, deben reunir, de cierta manera, conocimientos de índole pedagógica.

En cuanto a la "acreditación" de habilidades, debieran verificarse por la destreza misma, por ejemplo, con pruebas de nivel de competencia, a diferencia de ser acreditadas por el historial educativo de los alumnos.

Por último, también propone la forma de financiar a los profesores, y esboza tres posibilidades:

1. Mediante la institucionalización de centros de habilidades, de acceso libre y abierto al público.

2. Otorgar a ciertos grupos monedas educativas para asistir a centros en los cuales los demás clientes debieran pagar tarifas comerciales.

3. La propuesta más radical, mediante la creación de un banco para el intercambio de habilidades, con un crédito para adquirir las habilidades fundamentales, y por encima de eso, se otorgarían créditos adicionales a quienes lo ganasen enseñando. Para que este sistema resulte equitativo, propone un crédito diferencial para los más desfavorecidos.

El tercer canal es el servicio de búsqueda de compañero, que consiste en un sistema de comunicación que permita que una persona especifique la actividad por la cual busca un compañero y el sistema le devuelva los nombres y direcciones de aquellas personas que hubiesen introducido la misma descripción. Este sistema sería de utilidad para quienes quieran compartir o practicar una habilidad o determinado conocimiento. Illich utiliza la siguiente metáfora para ilustrarlo "A un buen jugador de ajedrez siempre lo contenta hallar un buen adversario, y aun novato le alegra el hallar otro" (p. 127), y sostiene que un sistema así invita a las personas, desde que son pequeñas, a conocer, evaluary buscar a otros y los prepararía para mantener a lo largo de toda la vida el interés por la búsqueda de nuevos socios para nuevos emprendimientos.

Además, plantea la necesidad de desescolarizar o desinstitucionalizar los grupos por edades, sexos y clase, así como abolir la obligatoriedad de asistir a reuniones y reconocer el derecho a la libre reunión. Para que esto suceda el sistema debiera ser público para garantizar la libre reunión. Las reuniones podrían llevarse a cabo en los edificios donde funcionan las escuelas actuales y ser gestionadas por la comunidad. El acceso a esos centros y reuniones debiera ser gratuito, o bien, pago mediante bonos educacionales (vouchers).

En cuanto al funcionamiento de la red de comunicación de búsqueda de compañero, llega a describir cómo sería el acceso, el almacenamiento y el procesamiento de la información, tanto desde el correo postal al sistema computacional, mediante terminales conectadas a una computadora capaces de brindar resultados inmediatos a la búsqueda de compañero, o bien a través de tablones de anuncios si no se encuentra compañero.

El cuarto canal propuesto es el de servicios de referencia respecto de educadores independientes. Este consiste en una especie de catálogo de educadores, profesionales o paraprofesionales independientes, que se postulan como tales, en el que se indicarían sus direcciones, descripciones y las condiciones de acceso a sus servicios. "Podría considerarse a tales redes como servicios de utilidad pública que permitiesen a los estudiantes elegir a sus profesores o a los pacientes sus médicos" (Illich, 1985, p. 134).

Se entiende por educador independiente a quien posea el conocimiento de una disciplina y sabiduría práctica, capaz de ofrecer una dirección intelectual al que se inicia en el aprendizaje. Según el propio Illich, los límites entre el profesor de habilidades (descrito en la sección sobre las lonjas de habilidades) y el líder educativo son siempre confusos. El papel de este último se fundamenta en una disciplina intelectual y una imaginación superiores. Debe ser capaz de corregir errores de conceptos y servir de guía en la exploración intelectual.

Este servicio será más necesario a medida que se ponga en marcha la trama de oportunidades, puesto que cuantas mayores posibilidades se tengan de elegir, la necesidad de buscar directivos críticos tendería a aumentar. Para Illich, la desescolarización invita a ampliar la búsqueda de educadores independientes dispuestos a guiar a quien inicia la tarea de educarse.

Para hacer posibles estas lonjas educacionales se requiere de dos tipos de "educadores independientes". Por un lado, quienes poseen conocimientos acerca del aprendizaje humano, y por el otro, aquellos que poseen conocimientos de una disciplina adquiridos durante toda la vida. En las escuelas, esos dos roles se funden en uno solo: el docente, y quienes poseen solo el segundo son vistos allí con malos ojos. Para Illich, a medida que se comprenda el nuevo funcionamiento de esta red de aprendizaje, habrá mayor demanda de 
educadores, y mayor oferta, puesto que ciertos docentes podrán aportar su conocimiento pedagógico. Los roles del "educador natural" y del "pedagogo" resultan complementarios y distintos, y lo ejemplifica de la siguiente manera:

Si un estudiante quisiese aprender cantonés hablado de un vecino chino, el pedagogo estaría a mano para juzgar el aprovechamiento y pericia de ambos, y para ayudarles a elegir el libro de texto y los métodos más adecuados para sus talentos, caracteres y tiempo disponible para estudiar. (Illich, 1985, p. 137).

Además, para un buen funcionamiento de la trama planteada aquí, se requiere un tercer rol, el del administrador, que más que un administrador de escuelas, encargado de supervisar, vigilar y registrar, se parece a un curador de museo, capaz de crear, manejar y facilitar el acceso a las redes educativas. Para comprender a cabalidad este canal y su función dentro de la trama de oportunidades, las palabras de Illich (1985) lo ilustran así:

\section{[...] los padres y otros "educadores naturales" nece- sitan un guía, las personas que aprenden necesitan ayuda, y las redes necesitan personas que las hagan funcionar. Los padres necesitan orientación para dirigir a sus hijos por el camino que conduce a la independencia educativa responsable. Los apren- dices necesitan líderes experimentados cuando se topan con un terreno arduo. Estas dos necesidades son muy distintas: la primera es una necesidad de pedagogía, la segunda una necesidad de dirección intelectual en todas las demás ramas del conocimien- to. (p. 135).}

Como puede verse, Illich no propone abolir toda asimetría sino aquellas que configuran formas autoritarias y jerárquicas de relación: las asimetrías funcionales parecen conformar un elemento indispensable en la lógica de cualquier aprendizaje humano.

\section{La pansofía por otros medios}

En ese escenario de debate, en el momento de su difusión, las propuestas de Illich recibieron no pocos elogios aunque también sufrieron unas cuantas críticas, algunas muy duras. Tal vez el punto más vulnerable de su postura era de carácter metodológico: aun quienes simpatizaban con su radical posición sospechaban del hecho de que simples reuniones interpersonales articuladas según grupos de interés habrían de ser, efectivamente, impracticables a gran escala, incapaces de sustituir nada menos que a fenomenales sistemas educativos nacionales bien organizados, estatalmente establecidos y legítima y legalmente consolidados, al menos en los inicios de la década de 1970. A los estudios de Ivan Illich se les adjudicaba mucha ingenuidad.

Posteriormente a la publicación de Deschooling Society, el propio Illich fue crítico de su obra. Al poco tiempo de publicada y a raíz de las críticas que recibió, realizó otro acercamiento a la noción moderna de educación y más tarde llegó a calificarla como ingenua, reconociendo que sus intereses en aquel momento estaban más cercanos a los efectos sociales que a la esencia de la educación (Bruno-Jofré y Zaldívar, 2016).

Respecto de los sectores sociales más vulnerables, aparecían en la mirada de la crítica a Illich como los más perjudicados por la propuesta, por poseer menos recursos para agenciar saberes por fuera de la organización escolar. Esta limitación metodológica, argumentaban, se vuelve en contra de la propia intencionalidad democratizadora de Illich (Gintis, 1975).

A pesar de estas críticas y de los desarrollos illichianos posteriores, su observación original de la escolarización y los cambios que propone en las relaciones entre enseñantes y aprendientes, así como en los recursos educacionales, permite un análisis de los dispositivos de la escuela moderna puestos en jaque por La sociedad desecolarizada. Este texto mantiene un valor central y, de hecho, con los años logró contravenir a no pocos de sus objetores, incluso al propio autor.

La primera de las objeciones parece haber sido en gran medida quebrantada por estos últimos treinta años de desarrollos tecnológicos, cambios en los regímenes de saber, abaratamiento de computadoras, facilidad para operar en pantallas, multiplicación de redes. Por ejemplo, el deseo de Illich de desescolarizar algunos artefactos cognitivos o de poner bibliotecas y centros de documentación al alcance de todos ya ha ocurrido en gran medida y las posibilidades de diseminación han sido tan rotundas que hoy nos hallamos con el problema inverso: cómo identificar lo más conveniente y cómo definirlo en forma genuina sin que esté mediatizado implícitamente por intereses políticos o comerciales ajenos a la misma búsqueda.

Respecto de la segunda objeción, no parecen quedar dudas de que el sistema propuesto por Ivan Illich no abandona la pansofía sino que supone alcanzarla por otros medios: por un lado, las escuelas ya no parecen efectivamente capaces de alcanzar este postulado universal; por otro, la pansofía solo puede mantener su vigencia si, justamente, se sacrifica la escuela a favor de disponer de recursos que por su propia dinámica la neutralizan: elementos organizados de manera tal que permitan una relación recíproca, convivencial, entre los que aprenden. Una relación que permita ir tejiendo una trama de aprendizajes a partir de la motivación personal y las oportunidades brindadas 
por cada uno de esos recursos y en interacción constante, lo que permitiría a cada uno diseñar su propio trayecto educativo, con el apoyo, el acompañamiento y la guía de otros.

Esta utopía podría parecer poco menos que delirante en los años setenta del siglo $\mathrm{xx}$, o ingenua incluso para un Illich más "maduro". Pero hoy suena plausible en términos técnicos. Está claro que la descripción de las acciones educacionales previstas por Illich resuenan a redes de computadoras hoy existentes y a acciones que de hecho ya están emprendidas, funcionan cotidianamente y hasta son gratuitas en un espacio público que Illich había propuesto pero nunca imaginado: el espacio virtual.

Las tramas de aprendizajes y las herramientas para la convivencialidad propuestas por Illich anticiparon el avance de internet (Kahn y Kellner, 2007; Waks, 2014). Las pantallas, las redes y la web 2.0 están cambiando las formas de acceder al conocimiento y permiten nuevas relaciones en torno al saber, aunque más fuera de la escuela que dentro de ella.

Esto podría entenderse dados los avances tecnológicos: se destacan propuestas pedagógicas que piensan los modos de articular nuevos entornos de aprendizaje capaces de adaptarse a las necesidades, los contextos culturales y los gustos de cada individuo, como respuesta al hecho de que las instituciones educativas modernas no están pudiendo responder al desafío que presenta la nueva generación de herramientas tecnológicas (Zaldívar y Castillo, 2015). La potencia argumentativa de Ivan Illich permite un análisis superador: no son las "herramientas tecnológicas" las que ponen en cuestión a las instituciones escolares, sino las relaciones sociales que las producen y las resignifican. Lejos de las miradas que ensalzan y reifican a la tecnología, son las relaciones sociales las que permiten imaginar escenarios en donde se pueda educar de otro modo.

Por otro lado, las críticas actuales a la escolarización unidas a la certeza de su carácter contingente, ya descrito, permite imaginar que sobre el piso de la mutación tecnológica se desplegarán relaciones sociales e intergeneracionales (relaciones convivenciales) que apenas nos atrevemos a imaginar.

Sabemos que las escuelas imaginadas por Comenius (1986) y diseñadas por sus textos en el siglo xvii demoraron varios siglos en materializarse y solo se están concretando en la actualidad, paradójicamente, durante el momento de mayor crítica a la vigencia de su organización. Como mostrara Eric Hobsbawm (2010), el diseño de una tecnología eficaz no es condición suficiente para su aplicación eficaz: hace falta un nuevo actor social capaz de reconfigurar lo viejo de una manera tan radicalmente original que así, y solo así, podrá implementar lo nuevo.

Es posible que, como el de Comenius, ese sea el destino de los textos y las ideas de Ivan Illich: que conformen una herramienta determinante en el momento en que las críticas a las escuelas se hayan sosegado para dar paso a su reformulación en algo completa y radicalmente distinto.

\section{Referencias}

Borges, J. y Ferrari, O. (2005). En diálogo I. Buenos Aires: Siglo XXI.

Bruno-Jofré, R. y Zaldívar, J. (2016). La crítica tardía de Ivan Illich a la sociedad desescolarizada: "estaba ladrando al árbol equivocado". Entramados-Educación y Sociedad, 3(3)65-81.

Burrell, G. y Morgan, G. (1979). Sociological paradigms and organizational analysis (248). Londres: Heinemann.

Comenius, J. (1986). Didactica Magna, Madrid: Akal.

Cuban, L. (1990). Reforming again, again, and again. Educational Researcher, 19(1), 3-13.

Durkheim, E. (1973). Education et Sociologie. París: puf.

Freire, P. (2005). Pedagogía del oprimido. Buenos Aires: Siglo xxi.

Gabbard, D. y Stuchul, D. (2016). Ivan Illich (1926-2002). En: J. Palmer Cooper y D. Cooper (eds.). The Routledge encyclopedia of educational thinkers. Nueva York: Routledge.

Gajardo, M. (1999). Ivan Illich. Perspectiva, 24, 808-821.

Gintis, H. (1975). Crítica de Ivan Illich. Crítica del illichismo: a propósito de la sociedad desescolarizada. Anagrama: Barcelona.

Hamilton, D. (2013). Towards a theory of schooling. Nueva York: Routledge.

Hobsbawm, E. (2010). Age ofrevolution: 1789-1848. Londres: Hachette, UK.

Illich, I. (1974). La convivencialidad. Barcelona: Barral Editores.

Illich, I. (1985). La sociedad desescolarizada. México: Barral Editores.

Kahn, R. y Kellner, D. (2007). Paulo Freire and Ivan Illich: Technology, politics and the reconstruction of education. Policy Futures in Education, 5(4), 431-448.

Lyotard, J. (1987). La condición posmoderna. Madrid: Cátedra. 
Martínez, A. y Narodowski, M. (1986). Escuela, historia, poder. En: Martínez Boom, A. y Narodowski, M. Escuela, historia y poder. Miradas desde América Latina. Buenos Aires: Novedades Educativas.

Narodowski, M. (1994). Infancia y poder. La conformación de la pedagogía moderna. Buenos Aires: Aique.

Narodowski, M. (1999). Después de clase. Buenos Aires: Novedades Educativas.

Narodowski, M. (2014). Infancia, pasado y nostalgia. Cambios en la transmisión intergeneracional. Revista Brasileira de História da Educação, 14(2), 35.

Narodowski, M. (2016). Un mundo sin adultos. Buenos Aires: Debate.
Valle, R.; Normandeau, S. y González, G. (2015). Education at a glance interim report: update of employment and educational attainment indicators. París: OCDE.

Waks, L. (2014). Education 2.0: The learning-web revolution and the transformation of the school. Boulder: Paradigm.

Zaldívar, J. y Castillo, X. (2015). Las teorías de la desescolarización y su continuidad en la pedagogía líquida del siglo xxi. Educación xxi, 20(1).

Zaldívar, J. (2016). Desescolarizar la vida. Ivan Illich y la crítica de las instituciones educativas. Madrid: Enclave de Libros. 